Article

\title{
Does Far-Infrared Therapy Improve Peritoneal Function and Reduce Peritonitis Recurrent in Peritoneal Dialysis Patients?
}

\author{
Yuanmay Chang ${ }^{1, *}$, Jui-Ting Chang ${ }^{2}$, Mei-Yi Lee ${ }^{3}$, Mei-Zen Huang ${ }^{4}$, Yann-Fen C. C. Chao ${ }^{5}$, Yung-Luen Shih ${ }^{1}$ and \\ Yao-Rong Hwang ${ }^{6}$ \\ 1 Institute of LongTerm Care, MacKay Medical College; E-mail: hwangyr@mmc.edu.tw \\ 2 Division of Nephrology, Department of Internal Medicine, Shin Kong Wu Ho-Su Memorial Hospital, Taipei, \\ Taiwan; Email: skhnephropgy@gmail.com \\ 3 Department of physical therapy, Fooyin University, Kaohsiung, Taiwan; Michelin54321@hotmail.com \\ 4 Department of nursing, National Tainan Junior College of Nursing, Tainan, Taiwan; E-mail: \\ meizen.huang@gmail.com \\ 5 Department of nursing, Hungkuang University, Taichung, Taiwan; E-mail: yannfen.chao@gmail.com \\ 6 Department of Pathology \& Laboratory Medicine, Shin Kong Wu Ho-Su Memorial Hospital, Taipei, Taiwan; \\ E-mail: t005524@ms.skh.org.tw \\ * Correspondence: r000085@mmc.edu.tw; Tel.: +886-919991261
}

\begin{abstract}
The treatment of peritoneal dialysis in end-stage renal disease is increasing in clinical practice. The main purpose of this study was to evaluate the effect of far-infrared radiation therapy on inflammation and the cellular immunity of patients undergoing peritoneal dialysis.

We recruited 56 patients undergoing peritoneal dialysis, and we included 32 patients from the experimental group and 24 patients from the control group in the final analysis. The experimental evaluation in our study was as follows: (1) We used abdominal computed tomography to measure the diameter and degree of hardening of the abdominal veins and large blood vessels to explore the changes in abdominal blood vessels. (2) The study compared the effects of peritoneal dialysis using albumin, blood urea nitrogen, creatinine, white blood cell, neutrophil-to-lymphocyte ratio, platelet-to-lymphocyte ratio, erythrocyte sedimentation rate, Hs-CRP clearance rate, and HBAIC. (3) We compared the cytokine concentration of blood between the two groups while controlling for GM-CSF, IL-2, IFN- $\gamma$, IL-6, IL-18, IL-4, IL-5, IL-13, IL-12p70, TNF- $\alpha$, and IL-1 $\beta$.

Results and Discussion: (1) There was no significant difference in the abdominal blood vessels of the experimental group relative to the control group according to abdominal CT measured over 6 months. (2) Our study demonstrates the statistically significant effect of FIR therapy on the following parameters: albumin $\left(p=0.048^{*}\right)$, creatinine $\left(p=0.039^{*}\right)$, and Hs-CRP $\left(p<0.001^{* *}\right)$ decreased significantly, and glomerular filtration rate (eGFR, $\left.p=0.043^{*}\right)$ and glucose $\left(p<0.001^{* *}\right)$ increased significantly. Our study found that, in the experimental group, albumin and creatinine decreased significantly due to FIR therapy for 6 months. However, our study also found that, in the experimental group, glucose $\left(\mathrm{p}<0.001^{* *}\right)$ increased significantly due to FIR therapy for 6 months. Peritoneal dialysis combined with FIR can reduce the side effects of glucose in dialysis buffer, which interferes with peritoneal inflammation and peritoneal mesothelial cell fibrosis. In addition, we also found that, in the experimental group, Hs-CRP $\left(p<0.001^{* *}\right)$ decreased significantly due to FIR therapy for 6 months. (3) No statistical significance in the inflammatory cytokines related to FIR therapy differences was observed in our study. IFN- $\gamma(p=0.124), I L-12 p 70(p=0.093)$, IL-18 $(p=$ $0.213)$, and TNF- $\alpha(\mathrm{p}=0.254)$ did not exhibit significant improvements in peritoneal dialysis with FIR treatment over 6 months. IFN- $\gamma$ and IL-18 in the plasma of patients in the experimental group and the control group were higher in the third month than in the first month.

Conclusion: We found that the effect of peritoneal dialysis improved significantly with FIR therapy, and significant improvements in the peritoneal permeability and inflammatory response were observed.
\end{abstract}

Keywords: end-stage renal disease; peritoneal dialysis; far-infrared therapy (FIR) 


\section{Introduction}

The treatment of peritoneal dialysis in end-stage renal disease (ESRD) is increasing in clinical practice. In Taiwan, diabetes is the main cause of kidney disease, and as many as $40 \%$ of dialysis patients suffer from nephropathy due to diabetes [1,2]. Relevant studies have confirmed that peritoneal dialysis is the favorable treatment for end-stage renal disease; however, the biggest limitation is that, as the peritoneal dialysis stage increases dialysis time, the peritoneum denatures, resulting in diffusion and convective transport, causing loss of ultrafiltration ability, and peritoneal dialysis [3]. In clinical studies, it is reported that the main reason for peritoneal dialysis failure is complications due to peritoneal inflammation. In patients with end-stage nephropathy, the immunity of the peritoneal cavity is reduced [4], resulting in conditions such as long-term peritoneal dialysis, uremia, repeated peritoneal infections, dialysis buffer, low $\mathrm{pH}$, and sugar decomposition products. All of these can induce inflammation and cause peritoneal mesothelial cell destruction, triggering the accumulation of monocytes, mast cells, and fibroblasts [5]. Moreover, these factors can also cause interstitial cell degeneration and fibrosis, fibrous deposition, vascular proliferation, plasma coagulation factor, and fibrin infiltration into the abdominal cavity, thereby reducing dialysis ultrafiltration rate [6].

Glucose is the most commonly used osmotic agent in peritoneal dialysis solutions. The continuous absorption of dextrose leads to hyperglycemia, exacerbation of diabetes mellitus (DM), hyperlipidemia, obesity, malnutrition, and titration of insulin dose in DM patients. Hyperglycemia can cause changes to the renal cell structure and glomeruli increase, which induces glomerular proliferation, glomerular basement membrane thickening, and fibrosis [7]. Glucose degradation products (GDPs) contribute to reduced dialysis function and the pathogenesis of peritoneal membrane fibrosis $[8,9,10]$. A previous study showed that long-term exposure to non-biocompatible high-concentration glucose dialysate and repeated peritonitis are the main causes of changes in the structure and function of the peritoneum $[4,11,12]$. In summary, these factors induce proinflammatory hormones, such as interleukin-l beta (IL-1 $\beta$ ), interleukin(IL), and tumor necrosis factor alpha (TNF- $\alpha$ ), which triggers a persistent inflammatory response in the peritoneum, ultimately leading to structural changes in the peritoneum $[13,14,15,16]$.

Far-infrared rays have been widely used in clinical healthcare and rehabilitation since 1800. Certain studies found far-infrared can vibrate the water molecules in our body to produce energy $[17,18]$. Far-infrared rays are called biological waves, and the effects produced are called biological effects $[19,20]$. The energy produced is divided into two categories: thermal effect and non-thermal effect, according to the form the energy takes [21]. Akasaki et al. (2006) found that FIR promotes angiogenesis in mice through a non-thermal effect. FIR was able to increase skin blood flow in rats [22, 23], and FIR inhibited interleukin-6 and TNF- $\alpha$ activity in mice with peritonitis [24]. In recent years, more studies confirmed and elucidated the effects of far-infrared rays in the human body. For example, FIR has been shown to promote blood circulation [23, 25], relieve fatigue and pain [26, 27], and promote wound healing [28,29,25,30].

As regards to the non-thermal effects, studies show that far-infrared rays can promote cell proliferation and wound healing [31,32], and can increase the expression of arterial endothelial nitric oxide synthase and nitric oxide synthesis in hamsters [33]. Moreover, it can promote angiogenesis in mice [22], and inhibit interleukin- 6 and TNF- $\alpha$ activity in mice with peritonitis [24]. Far-infrared radiation therapy can promote hemeoxygenase-1 (HO-1) and endothelial nitric oxide synthase (eNOS) generated by the L-Arginine/nitric oxide pathway, reducing inflammation through the inhibition of intimal hyperplasia and reducing oxidation. It can even stimulate the inhibition of tumor necrosis factor $\alpha$ (TNF- $\alpha$ ) to produce anti-inflammatory effects [34, 29, 32, 35, 36, 23, 37]. Although the mechanisms involved in FIR are not clear, various studies indicate that far-infrared rays can promote the proliferation of bone marrow stem cells and keratinocytes through the CXCR4/ERK or Notch1/Twist pathways [38, 31, 39, 40]. Excessive oxi- 
dative stress and inflammation are the major factors that promote abnormal cell proliferation. Lin et al. (2013) found that far-infrared rays can enhance the activity of vascular endothelial cells and promote angiogenesis [41], and can inhibit inflammation by inhibiting inflammatory factors, such as IL-6 and TNF- $\alpha$ [42, 24]. Furthermore, they can improve mitochondrial function effects [43, 44]. Although how far-infrared therapy affects inflammation and cellular immunity in patients undergoing peritoneal dialysis remains to be explained, various studies indicate that IL-4 and IL-13 activated macrophages play critical roles in the resolution of inflammation and the restoration of tissue homeostasis $[45,46,47,48,49,50]$.

The literature concerning the effect of far-infrared radiation therapy on inflammation in patients undergoing peritoneal dialysis is limited. Therefore, in this study, we aimed to study the physiological condition and inflammatory response of dialysis patients who had been irradiated with far-infrared rays for 6 months. The main purpose of this study was to evaluate the effect of far-infrared radiation therapy on inflammation and the cellular immunity of patients undergoing peritoneal dialysis.

\section{Materials and Methods}

\subsection{Research Objects}

In this study, we selected patients with the following conditions from the peritoneal dialysis area of the Shin Kong Wu Ho-Su Memorial Hospital medical center, Taipei:

(1) Patients with peritoneal dialysis stabilized for 3 months.

(2) Patients with no peritonitis, with a low dialysis fluid and plasma creatinine ratio $(\mathrm{D} / \mathrm{P} \mathrm{Cr}$ ) and peritoneal permeability, who did not need to use hypertonic dialysate, and with a C-reactive protein (CRP) index that did not increase.

(3) Patients under 75 years old who could perform peritoneal dialysis fluid exchange alone or with the assistance of a family member.

\subsection{Patient Selection}

The study was conducted according to the guidelines of the Declaration of Helsinki, and was approved by the Institutional Review Board of the medical center. There were 151 patients on peritoneal dialysis in the medical center, and 71 of these met the conditions. Two patients died, two patients underwent kidney transplantation, and 11 patients were hospitalized without FIR and so withdrew from this study over the 6 months. In this study, 56 patients on peritoneal dialysis were randomly assigned to the experimental group (32) and the control group (24). According to the literature, patients who underwent kidney transplantation or were hospitalized during the study period were excluded from the study, because of an inability to assess the far-infrared ray therapy. In order to perform a complete far-infrared radiation study, the observation period was at 3 months to 6 months (Figure 1). 


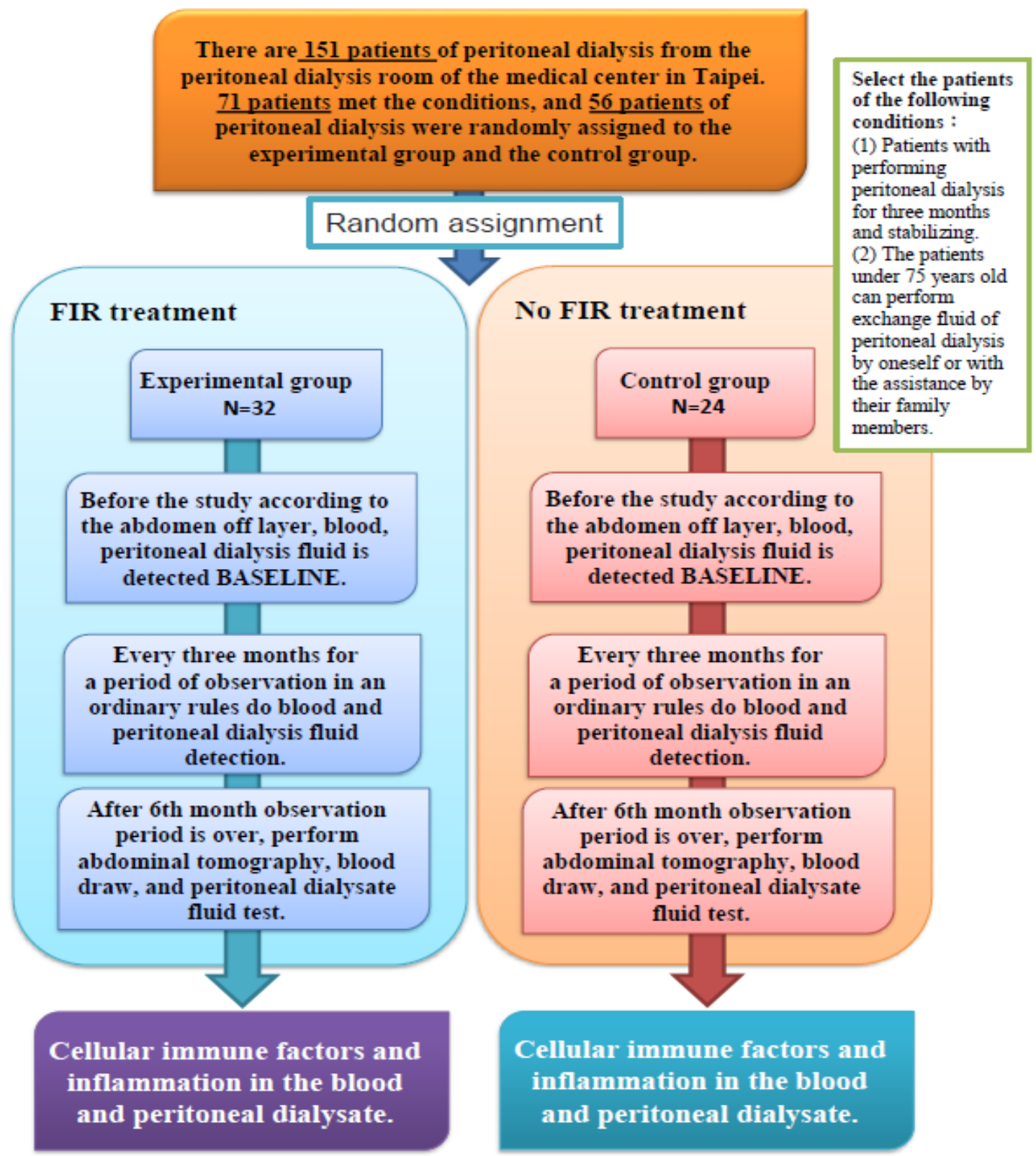

Figure 1. Flow chart of participants comparing the effect of patients undergoing FIR treatment and non-FIR (far-infrared) treatment.

\subsection{Experiment Method}

Before going to bed, the peritoneal dialysis patients had their fluid emptied and replaced each day; it was then irradiated for 40 minutes after the fluid input. The patients drew blood each third month, and the data from the 6-month period were assessed.

\subsection{Experimental Disposal (FIR instrument)}

This study used the WS TY301 FIR Emitter (WS Far-Infrared Medical Technology, Taipei, Taiwan) far-infrared instrument, which has a wavelength of $3 \sim 25 \mu \mathrm{m}$, and a peak of $8 \mu \mathrm{m}[51,52,53]$. The treatment location was centered on the navel, i.e., the entire abdominal cavity, from the lower edge of the sternum to the groin. The distance between the far-infrared instrument and the skin was $25 \sim 30 \mathrm{~cm}[51,52,53]$. Before going to bed, the peritoneal dialysis patients had their fluids emptied and replaced each day; it was then irradiated for 40 minutes using the far-infrared instrument (WS TY301 FIR emitter) after the fluid input. The patients in the other group did not receive far-infrared radia- 
tion treatment and served as the control group. The dialysis effect of patients in the control group was compared with that of the experimental group after irradiating the abdomen with far-infrared rays for 6 months.

\subsection{Centrifugal Collection of Plasma}

(1) Plasma collection: The CBC and DC of blood collection tubes contained EDTA, and the plasma was centrifuged at $800 \mathrm{~g}$ for 15 minutes. The upper supernatant of the plasma was pipetted equally into $110 \mu \mathrm{L}$ Eppendorf tubes. The remaining white blood cells in the Buffy coat were collected into another tube, and hemolysis was performed using erythrocytes lysis buffer to remove erythrocytes without a cell nucleus. A total of $0.4 \mathrm{~mL}$ of solution with white blood cells was separated equally into $200 \mu \mathrm{L}$ Eppendorf tubes to collect RNA and DNA.

(2) Determination of immune factors in plasma: The plasma, peritoneal dialysate, and the associated supernatant were used for multiple analysis of cytokines using Human Th1/Th2 Panels of Luminex 200 (BioRad Corporation, Madison). We aimed to analyze the release innate immunity cytokines after peritoneal dialysis patients had been irradiated with FIR, including GM-CSF, IL-2, IFN-gamma

(IFN- $\gamma$ ), IL-6, IL-18, IL-4, IL-5, IL-13, IL-12p70, TNF- $\alpha$, and IL-1 $\beta$, in order to confirm the effects of the inflammatory response in peritoneal dialysis patients [54].

\subsection{Experimental Evaluation}

1. The study used abdominal computed tomography (CT) to measure the diameter and degree of hardening of the abdominal veins and large blood vessels to explore the changes in the abdominal blood vessels [53]. The checkpoints were as follows: before the FIR instrument was used; the third month (after the FIR instrument was used for 3 months), and the sixth month (after the FIR instrument was used for 6 months). The dialysis effect and the degree of abdominal vascular stiffness in the experimental group and the control group were measured.

2. The study compared the effects of peritoneal dialysis using Bun, Creatinine $(\mathrm{Cr})$ value, and the dialysate and plasma creatinine ratio $(\mathrm{D} / \mathrm{P} \mathrm{Cr})$ from the blood and the peritoneal dialysate (the emptied dialysate fluid) every 3 months.

After the blood and peritoneal dialysate were collected, the blood hemoglobin, albumin (ALB), blood urea nitrogen (BUN), creatinine (Cr), white blood cell (WBC), neutrophil-to-lymphocyte $(\mathrm{N} / \mathrm{L})$ ratio, platelet-to-lymphocyte ratio, erythrocyte sedimentation rate, Hs-CRP clearance rate, and HBAlC (the first and the last time) analyses were used to assess check peritoneal inflammation, with the hope of slowing down the incidence of peritoneal dialysis using this treatment.

3. Evaluation of Serum Biochemical Parameters

The research process and the selection conditions were explained and the consent of the patients or family was obtained. A total of 56 peritoneal dialysis patients were selected, and each participant was randomly assigned to the experimental group or the control group. The experimental group (peritoneal dialysis patients), which received far-infrared abdominal irradiation for 6 months after dialysis, was compared with the control group. The research design is as follows (Figure 1).n order to observe the changes in plasma cytokines in peritoneal dialysis patients in the experimental group and the control group in different observation periods after far-infrared irradiation, plasma was collected in February (before irradiation), May (after irradiation for 3 months), and August (after irradiation for 6 months). The cytokines secreted by type 1 and type 2 helper T cells play an important role in infection. In this study, 11 kinds of cytokines related to type 1 and type 2 helper T cells were screened and analyzed, including GM-CSF, IL-2, IFN- $\gamma$, IL-6, IL-18, IL-4, IL-5, 
IL-13, IL-12p70, TNF- $\alpha$, and IL-1 $\beta$. We used repeated-measures ANOVA to compare the cytokine concentration of blood between the two groups, while controlling for GM-CSF, IL-2, IFN- $\gamma$, IL-6, IL-18, IL-4, IL-5, IL-13, IL-12p70, TNF- $\alpha$, and IL-1 $\beta$.

\subsection{Statistical Analysis}

The statistical analysis was performed using SPSS statistics (version 26, IBM SAS Institute, USA), and standard descriptive statistics were used to assess the baseline characteristics. Data are presented as mean \pm standard deviation. Repeated-measures ANOVA was performed to compare serial changes in the clinical data and echo parameters. $P$-values $<0.05$ were considered statistically significant, and $p$-values $<0.01$ were significantly different.

\section{Results}

\subsection{Patient Characteristics}

We recruited 71 patients undergoing peritoneal dialysis in this study. Of these, nine did not meet the inclusion criteria. As described above, we divided the remaining patients into groups according to the patient number: 39 patients were assigned to the experimental group and 32 patients were assigned to the control group. Therefore, we included 32 patients from the experimental group $(82.1 \%$ of the initial group) and 24 patients from the control group (75\% of the initial group) in the final analysis (Figure 1). The baseline demographic and clinical characteristics of the patients who completed the study did not differ significantly between the two groups.

\subsection{Evaluation of Abdominal Blood Vessels}

We used abdominal Computed Tomography (CT) to measure the diameter and degree of hardening of the abdominal veins and large blood vessels in order to explore the changes in abdominal blood vessels. The dialysis effect difference of the experimental group as compared to the control group did not reach statistical significance in abdominal blood vessels according to the CT scan at the three checkpoints (Table 1).

Table 1. Abdominal blood vessels of experimental group (FIR) and control group (non-FIR).

\begin{tabular}{cccc}
\hline Parameters & $\begin{array}{c}\text { Experimental group } \\
(\mathrm{n}=32)\end{array}$ & $\begin{array}{c}\text { Control group } \\
(\mathrm{n}=24)\end{array}$ & $\mathrm{p}$ \\
\hline Abdominal blood vessels $(\mathrm{nm})$ & $24.53 \pm 9.25$ & $25.31 \pm 10.19$ & 0.121 \\
\hline
\end{tabular}

Continuous variables were presented as mean \pm standard deviation. Categorical variables were presented as number (percentage). ${ }^{*}: p<0.05 ;{ }^{* *}: p<0.01$ by using SPSS statistics (IBM SAS Institute, USA) standard descriptive statistics.

\subsection{Physiological evaluation}

Using blood samples, we assessed all physiological and biochemical responses after baseline and 6 months of FIR intervention. In Table 2, it can be observed that the baseline demographic and clinical parameters between the two groups of patients were similar, excepting glucose $\left(p<0.001^{* *}\right)$, creatinine $\left(p=0.039^{*}\right)$, albumin $\left(p=0.048^{*}\right)$, eGFR $\left(p=0.043^{*}\right)$, and Hs-CRP $\left(p<0.001^{* *}\right)$. Even though no statistical significance in the blood urea nitrogen $(p=0.121)$ or WBC $(p=0.365)$ were found between the experimental group and the control group, we observed that the blood urea nitrogen increased in the control group and decreased in the experimental group over 6 months. We also observed that WBC decreased in the experimental group and increased in the control group over the 6 months. 
Table 2. Baseline demographic and clinical parameters of experimental group (FIR patients) and control group (non-FIR patients).

\begin{tabular}{|c|c|c|c|c|c|c|c|}
\hline \multirow{2}{*}{ Parameters } & \multicolumn{3}{|c|}{ Experimental group $(n=32)$} & \multicolumn{3}{|c|}{ Control group $(n=24)$} & \multirow{2}{*}{$\mathrm{p}$} \\
\hline & 0 & 3 & 6 & 0 & 3 & 6 & \\
\hline Age (years) & & \multicolumn{2}{|c|}{$54.53 \pm 12.56$} & \multicolumn{3}{|c|}{$58.31 \pm 8.19$} & \\
\hline \multicolumn{8}{|l|}{ Peritoneal function } \\
\hline Peritoneal Kt/V & \multicolumn{3}{|c|}{$1.96 \pm 0.28$} & \multicolumn{3}{|c|}{$2.02 \pm 0.21$} & 0.061 \\
\hline $\begin{array}{l}\text { Peritoneal weekly } \\
\text { CCr (L/week/ m2) }\end{array}$ & \multicolumn{3}{|c|}{$58.17 \pm 15.82$} & \multicolumn{3}{|c|}{$61.66 \pm 17.32$} & 0.123 \\
\hline \multicolumn{8}{|l|}{ Serum biochemistry } \\
\hline Glucose (mg/dL) & $105.27 \pm 29.48$ & $111.27 \pm 28.37$ & $99.40 \pm 12.25$ & $98.85 \pm 10.24$ & $99.31 \pm 9.75$ & $105.08 \pm 13.72$ & $<0.001^{* *}$ \\
\hline WBC & $6.51 \pm 2.26$ & $5.90 \pm 1.97$ & $6.68 \pm 2.24$ & $8.28 \pm 2.74$ & $7.59 \pm 1.84$ & $7.89 \pm 2.01$ & 0.365 \\
\hline RBC & $3.32 \pm 0.48$ & $3.26 \pm 0.50$ & $3.41 \pm 0.62$ & $3.45 \pm 0.55$ & $3.49 \pm 0.57$ & $3.54 \pm 0.61$ & 0.131 \\
\hline $\mathrm{HbA1c}(\%)$ & $9.93 \pm 1.51$ & $9.79 \pm 1.65$ & $10.21 \pm 1.91$ & $9.68 \pm 1.46$ & $10.22 \pm 1.69$ & $10.25 \pm 1.85$ & 0.757 \\
\hline BUN (mg/dL) & $63.67 \pm 14.77$ & $62.27 \pm 13.03$ & $61.13 \pm 12.22$ & $71.85 \pm 13.38$ & $64.15 \pm 20.40$ & $60.01 \pm 12.45$ & 0.42 \\
\hline Creatinine (mg/dL) & $9.07 \pm 3.48$ & $7.79 \pm 4.25$ & $6.23 \pm 0.68$ & $12.02 \pm 1.69$ & $10.22 \pm 1.69$ & $9.16 \pm 0.21$ & $0.039^{*}$ \\
\hline Albumin (g/dL) & $3.68 \pm 0.30$ & $3.57 \pm 0.38$ & $3.73 \pm 0.43$ & $3.56 \pm 0.42$ & $3.67 \pm 0.40$ & $3.73 \pm 0.56$ & $0.048^{*}$ \\
\hline eGFR & $4.21 \pm 1.41$ & $3.96 \pm 0.91$ & $3.73 \pm 0.78$ & $4.01 \pm 0.75$ & $3.99 \pm 0.73$ & $4.12 \pm 0.78$ & $0.043^{*}$ \\
\hline Phosphate (mg/dL) & $4.96 \pm 1.02$ & $4.83 \pm 0.91$ & $5.01 \pm 0.68$ & $5.13 \pm 1.43$ & $5.02 \pm 1.61$ & $5.36 \pm 1.12$ & 0.58 \\
\hline T-P & $6.52 \pm 0.71$ & $6.48 \pm 0.54$ & $6.47 \pm 0.66$ & $6.44 \pm 0.52$ & $6.47 \pm 0.58$ & $6.36 \pm 0.65$ & 0.314 \\
\hline Hs-CRP (mg/dL) & $1.31 \pm 2.14$ & $0.51 \pm 0.54$ & $0.75 \pm 1.37$ & $0.98 \pm 1.49$ & $1.00 \pm 2.21$ & $0.41 \pm 0.36$ & $<0.001^{* *}$ \\
\hline
\end{tabular}

Continuous variables were presented as mean \pm standard deviation. Categorical variables were presented as number (percentage). ${ }^{*}: p<0.05 ;{ }^{* *}: p<0.01$ by using Mann-Whitney U test (two-tailed)/Fisher's exact test. Kt/V: Kt/V urea; CCr: creatinine clearance; HbA1c: glycated hemoglobin; BUN: blood urea nitrogen; hs-CRP: high-sensitivity C-reactive protein.

\subsection{Evaluation of Serum Biochemical Parameters}

The results showed that there was no expression of GM-CSF, IL-2, IL-5, or IL-13 in the plasma of the experimental group or the control group. In Table 3, no statistical significance in the inflammatory cytokines related to FIR therapy differences in the experimental group or the control group was observed. We noted improvements in the variety of IL-6 $(p=0.061)$, IL-4 ( $p=0.156)$, IL-1 $\beta(p=0.175)$, and TNF- $\alpha(p=0.254)$, but these differences did not reach statistical significance (Table 3 and Figure 2). However, the plasma IL-18 levels in the experimental group and the control group were higher than those in the other two observation periods at the third month $(p=0.213)$ (Figure 2). A higher expression of IL-12p70 was detected in the plasma of patients in the experimental group at the third month, which lasted until the sixth month $(p=0.093)$ (Figure 2$)$. However, the plasma IFN- $\gamma$ of the experimental group decreased in the sixth month, recording a lower value than in the third month $(p=0.124)$ (Figure. 2). Although repeated-measures ANOVA showed that the pre-post values improved in the experimental group as compared to the control group, the difference was not statistically significant (Table 3). Repeated-measures ANOVA also revealed improvements in the variety of IFN- $\gamma$, IL-12, IL-18, and TNF- $\alpha$, but these differences did not reach statistical significance (Figure 2). 

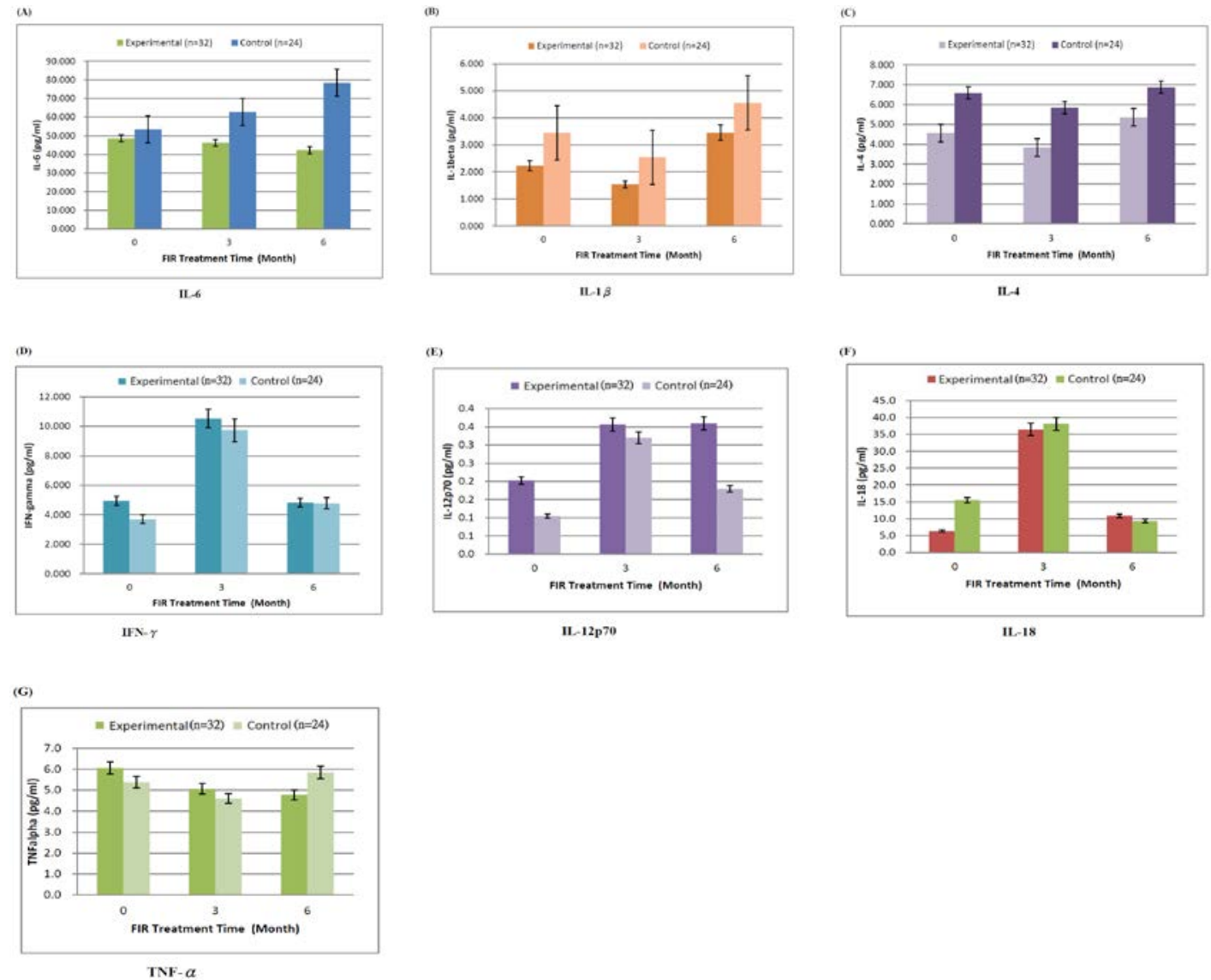

Figure 2. Comparison of biochemical clinical parameters of experimental group (FIR) and control group (non-FIR). (A) IL-6, (B) IL-1 , (C) IL-4, (D) IFN- $\gamma$, (E) IL-12p70, (F) IL-18, (G) TNF- $\alpha$.

Table 3. Biochemical evaluation and clinical parameters of experimental group (FIR) and control group (non-FIR).

\begin{tabular}{cccccccc}
\hline \multirow{2}{*}{ Parameters } & \multicolumn{3}{c}{ Experimental group (n= 32) } & \multicolumn{3}{c}{ Control group (n= 24) } \\
& $\mathbf{0}$ & $\mathbf{3}$ & $\mathbf{6}$ & $\mathbf{0}$ & $\mathbf{3}$ & $\mathbf{6}$ \\
\hline IL-1beta & $2.23 \pm 0.44$ & $1.54 \pm 0.14$ & $3.45 \pm 0.75$ & $3.45 \pm 0.39$ & $2.54 \pm 0.42$ & $4.55 \pm 1.12$ \\
IL-4 & $4.56 \pm 1.75$ & $3.84 \pm 0.87$ & $5.36 \pm 2.44$ & $6.58 \pm 1.98$ & $5.84 \pm 1.58$ & $6.87 \pm 2.12$ \\
IL-6 & $48.6 \pm 9.49$ & $46.2 \pm 12.35$ & $42.3 \pm 11.63$ & $53.5 \pm 11.64$ & $58.8 \pm 19.42$ & $65.4 \pm 21.75$ \\
IFN-gamma & $4.93 \pm 1.44$ & $10.52 \pm 2.86$ & $4.83 \pm 1.43$ & $3.69 \pm 0.94$ & $9.73 \pm 2.53$ & $4.78 \pm 2.14$ \\
IL-12p70 & $0.20 \pm 0.09$ & $0.36 \pm 1.14$ & $0.36 \pm 0.09$ & $0.175 \pm 0.04$ & $0.32 \pm 0.053$ & $0.18 \pm 0.07$ \\
TNF-alpha & $6.06 \pm 2.23$ & $5.07 \pm 2.12$ & $4.78 \pm 1.47$ & $5.39 \pm 2.18$ & $4.61 \pm 1.97$ & $5.85 \pm 2.11$ \\
IL-18 & $6.35 \pm 2.12$ & $36.48 \pm 8.9$ & $10.88 \pm 2.47$ & $15.51 \pm 4.23$ & $38.11 \pm 11.64$ & $9.34 \pm 3.11$ \\
\hline \multicolumn{2}{c}{ Continuous variables were presented as mean \pm standard deviation. Categorical variables were presented as number (percentage). } \\
\multicolumn{7}{c}{$* 0.051$} \\
\hline
\end{tabular}

\section{Discussion}

\subsection{Abdominal Vessels}

This study used abdominal computed tomography to measure the diameter and sclerosis of the abdominal veins and large vessels in order to explore the changes in abdominal vessels [53]. The dialysis effect in the experimental group relative to the control group exhibited no significant difference as a result of far-infrared radiation treatment for 6 months. This may be due to the short FIR time, the PD effect of patients not being completely collected, or the glucose concentration of the dialysate changing the dialysis effect. 


\subsection{The Physiological Effect of Peritoneal Dialysis Due to FIR}

Our study demonstrated the statistically significant effect of FIR therapy on the following parameters: $(1)$ albumin $\left(\mathrm{p}=0.048^{*}\right)$, creatinine $\left(\mathrm{p}=0.039^{*}\right)$, and Hs-CRP $(\mathrm{p}<$ $\left.0.001^{* *}\right)$ decreased significantly, and (2) the glomerular filtration rate (eGFR, $\mathrm{p}=0.043^{*}$ ) and glucose $\left(p<0.001^{* *}\right)$ increased significantly. Our study found that, in the experimental group, albumin and creatinine decreased significantly due to FIR therapy for 6 months. Creatinine is often used to assess the severity of renal insufficiency. In the experimental group, creatinine decreased significantly and the glomerular filtration rate increased significantly, indicating that far-infrared irradiation can improve the effect of peritoneal dialysis, which is in accordance with the literature [17, 41]. Despite no statistical significance in the blood urea nitrogen $(\mathrm{p}=0.121)$ between the experimental group and the control group being found, the blood urea nitrogen increased in the control group and decreased in the experimental group over the 6 months. Our study found that the blood urea nitrogen increased in the control group, which means that the effect of peritoneal dialysis decreased and urea nitrogen was accumulated in the blood without being excreted, resulting in an increase in the degree of renal function damage. We expected FIR to increase the peritoneal permeability and slow down the rate of peritoneal degeneration. The thermal effect of FIR treatment was vasodilation and increased vascular flow [17]. Lin et al. (2013) found that far-infrared rays can enhance the activity of vascular endothelial cells and promote angiogenesis [41]. The ability of microvascular endothelial cells to form tubes and move is also significantly improved after far-infrared radiation [55]. Therefore, we found that the effect of peritoneal dialysis was significantly improved with FIR therapy.

Our study also found that, in the experimental group, glucose $\left(p<0.001^{*}\right)$ increased significantly due to FIR therapy for 6 months. Long-term exposure to non-biocompatible high-concentration glucose dialysate and repeated peritonitis are the main causes of changes in the structure and function of the peritoneum [4]. The glucose concentration of different dialysis buffers may impact the burden of kidney filtration rate [11]. Studies have shown that the glucose concentration also promotes peritoneal inflammation and the production of transforming growth factor by peritoneal mesothelial cells, finally leading to fibrosis [12]. Therefore, peritoneal dialysis combined with FIR can reduce the side effects of glucose in dialysis buffer, which interferes with peritoneal inflammation and peritoneal mesothelial cell fibrosis. This finding is in accordance with the literature [11].

In addition, our study also found that Hs-CRP $\left(\mathrm{p}<0.001^{* *}\right)$ in the experimental group decreased significantly due to FIR therapy for 6 months. Hs-CRP acute reactive protein is a serum protein in the acute phase, which can be used as a good indicator of local inflammation or tissue injury [56]. Despite no statistical significance being observed in WBC ( $p=0.365)$ between the experimental group and the control group, we observed that WBC decreased in the experimental group and increased in the control group over the 6 months. Our results demonstrate that patients in the experimental group undergoing FIR therapy had significantly reduced inflammatory responses, and may have exhibited improved vascular endothelial cell function. Chang (2018) and Lee et al. (2019) also mentioned that FIR treatment can improve vascular endothelial cell function and help reduce the inflammatory response [24, 28]. Accordingly, we found that patients in our experimental group reported significant improvements in the inflammatory response [54].

\subsection{Inflammatory Cytokines}

In Table 3, it can be seen that no statistical significance in the inflammatory cytokines with FIR therapy differences was observed in our study. Originally, we hoped to see a similar situation to that revealed by Chang (2018) as regards to IL-6/TNF- $\alpha /$ INF- $\gamma$ [24]. In the present study, IFN- $\gamma(p=0.124)$, IL-12p70 $(p=0.093)$, IL-18 $(p=0.213)$, and TNF- $\alpha(p=$ 
0.254) did not exhibit significant improvements in peritoneal dialysis with FIR treatment for 6 months. However, as cytokines such as IL-6/TNF- $\alpha / \mathrm{INF}-\gamma$ are short-term inflammatory cytokines, we believe that the change in IFN- $\gamma$, IL-12, IL-18, and TNF- $\alpha$ can be classified as long-term inflammatory changes, according to the inflammatory situation in this study. TNF- $\alpha$ is mainly related to systemic inflammation [54]. As the TNF- $\alpha$ in the plasma of the subjects in the experimental group continuously decreased in the three observation periods, it was confirmed that the clinical inflammatory symptoms of the subjects improved due to the influence of far-infrared radiation treatment, which is similar to the physiological phenomenon in our study (i.e., Hs-CRP decreased significantly). However, IFN- $\gamma$ and IL-18 in the plasma of patients in the experimental group and the control group were higher at the third month than the first month. When macrophages are activated by an antigen, they secrete IL-18, and then chemotactic T cells secrete IFN- $\gamma$. Furthermore, IFN- $\gamma$ stimulate other macrophages or related immune cells to eliminate microbial pathogens [54]. TNF- $\alpha$ and IFN- $\gamma$ are vital symbols of cellular immunity and play an important role in immunotherapy, while IL-12 plays critical roles in the activation of innate immunity [54]. High IL-12p70 expression was observed in the plasma of patients in the experimental group at the third month, and this lasted until the sixth month. One of the effects of IL-12p70 is to stimulate the production of IFN- $\gamma$, which is anti-inflammatory. However, the IFN- $\gamma$ in the plasma of the experimental group decreased in the sixth month, recording a lower value than in the third month. Moreover, we found that patients in our experimental group reported improvements in the inflammatory response over the 6 months.

\subsection{Overall Assessment}

Although there was no significant difference in the inflammatory cytokines throughout the study, we found that patients in our experimental group reported improvements in the inflammatory response over the 6 months. Previous studies reported that the mechanism of far-infrared therapy is related to its non-thermal effects. The non-thermal effect promotes vascular endothelial proliferation by stimulating HO-1[35, 36, 23]. Lin et al. (2013) demonstrated that the stimulation of HO-1 expression leads to the suppression of TNF- $\alpha$ expression in hemodialysis patients [52]. Chang (2018) found that FIR treatment can inhibit or reduce the activity of IL- 6 and TNF- $\alpha$ and can stabilize the expression of eNOS in mice [24]. FIR therapy can also enhance HO-1 synthesis, activate eNOS, inhibit vascular endothelial growth, reduce oxidative vascular sclerosis, and improve the effect of vascular repair [24, 34, 57]. Chang (2018) and Lee et al. (2019) also mentioned that FIR treatment can reduce and delay IL- 6 and TNF- $\alpha$ responses, which may improve vascular endothelial cell function and help reduce the inflammatory response $[24,28]$. Although the changes in IFN- $\gamma$, IL-12, IL-18, and TNF- $\alpha$ were not statistically significant, patients in the experimental group reported improvements in the inflammatory response. These results indicate that far-infrared therapy may have a greater positive impact on blood circulation, and for this reason, the long-term effects of far-infrared therapy need to be further studied. In this study, the effect of FIR on the long-term integrity of the peritoneum, how it improved the peritoneal exchange material, and how it reduced the incidence of peritonitis in peritoneal dialysis patients were only studied over 6 months. It is thought that FIR can slow down the rate of peritoneal degeneration, increase the peritoneal permeability, and reduce the frequency of peritoneal inflammation. Therefore, further studies are needed to obtain a better understanding of the effects of FIR therapy on patients with peritoneal dialysis treatment.

\section{Conclusions}

In conclusion, our study demonstrated that the effect of peritoneal dialysis can be improved with FIR in terms of the physiological effects and the clinical inflammatory symptoms. 
Acknowledgements: This work was supported by a grant from Mackay Medical College in Taiwan. We thank the clinical staff of Shin Kong memorial hospital who referred the patients and collected blood samples. We also thank WS Far IR Medical Technology Co., Ltd., for technical support.

Funding: This research was funded by grants from the Academic Research of Mackay Medical College, grant number, 1041A01.

Institutional Review Board Statement: The study was conducted according to the guidelines of the Declaration of Helsinki and approved by the Institutional Review Board of the Shin Kong Wu Ho-Su Memorial Hospital (IRB No: 20150902R). This study was registered in the Thai Clinical Trials Registry (TCTR20211125005).

Informed Consent Statement: We obtained written informed consent from participants prior to the study. Written informed consent to publish this paper was obtained from the patients.

Data Availability Statement: Not applicable.

Conflicts of Interest: The authors declare no conflicts of interest.

\section{References}

1. Jamie, P.D.; Julia, B.L. Clinical aspects of diabetic nephropathy. In: Robert WS, Eric GN, eds. Schrier's Diseases of the Kidney. 19th. Lippincott Williams \& Wilkins. 2012, 165975.

2. Alicic, R.Z.; Rooney, M.T.; Tuttle, K.R. Diabetic Kidney Disease: Challenges, Progress, and Possibilities. Clin J Am Soc Nephrol. 2017, 12, 2032-45.

3. Diabetes Control and Complications Trial Research Group; Nathan, D.M.; Genuth, S.; Lachin, J.; Cleary, P.; Crofford, O.; Davis, M.; Rand, L.; Siebert, C. The effect of intensive treatment of diabetes on the development and progression of long-term complications in insulin-dependent diabetes mellitus. N Engl J Med. 1993, 329, $977-86$.

4. Yang, X.; Yu, XQ. Approaches to the protection of peritoneum in patients on long-term peritoneal dialysis. Chinese Journal of Blood Purification, 2010, 9 (10), 523-525.

5. Muskiet, M.H.; Smits, M.M.; Morsink, L.M.; Diamant, M. The gutrenal axis: do incretin-based agents confer renoprotection in diabetes. Nat Rev Nephrol. 2014, 10, 88-103.

6. Writing Team for the Diabetes C, Complications Trial/Epidemiology of Diabetes I, Complications Research G. Effect of intensive therapy on the microvascular complications of type 1 diabetes mellitus. JAMA. 2002, 287, 2563-9.

7. Fineberg, D.; Jandeleit-Dahm, K.A.; Cooper, M.E. Diabetic nephropathy: diagnosis and treatment. Nat Rev Endocrinol. 2013, 9, 713-23.

8. Sowinski, K.M.; Churchwell, M.D.; Decker, B.S. Hemodialysis and Peritoneal Dialysis. In Pharmacotherapy: A Pathophysiologic Approach, 11th ed., DiPiro, J.T.; Yee, G.C.; Posey, L.M.; Haines, S.T.; Nolin, T.D.; Ellingrod, V. Eds.; McGraw-Hill Education: New York, NY, USA, 2020.

9. Mehrotra, R. Metabolic Effects of Peritoneal Dialysis. In Handbook of Dialysis Therapy, 5th ed., Nissenson, A.R.; Fine, R.N. Eds., Elsevier: New York, NY, USA, 2017.

10. Wieslander, A.P.; Nordin, M.K.; Martinson, E.; Kjellstrand, P.T.; Boberg, U.C. Heat Sterilized PD-Fluids Impair Growth and Inflammatory Responses of Cultured Cell Lines and Human Leukocytes. Clin. Nephrol. 1993, 39, 343-348.

11. Chang, C.N.; Niu, C.Y.; Tan, A.C.; Chan, C.H.; Chen, C.F.; Chen, T.H.; Li, S.Y.; Chen, Y.T.; Chen, F.Y.; Liu, W.S.; Lin, C.C.; Wei, G.J. The Effect of Far-Infrared Therapy on the Peritoneal Expression of Glucose Degradation Products in Diabetic Patients on Peritoneal Dialysis. Int J Mol Sci. 2021, 22(7), 3732.

12. Jiang, S.K.; Feng, S.; Shi, Y.B. Association between serum leptin levels and peritoneal dialysis: A meta-analysis. Experimental and Therapeutic Medicine, 2015, 10(1), 300-308. 
13. Witowski, J.; Wisniewska, J.; Korybalska, K.; Bender, T.O.; Breborowicz, A.; Gahl, G.M.; Frei, U.; Passlick-Deetjen, J.; Jörres, A. Prolonged Exposure to Glucose Degradation Products Impairs Viability and Function of Human Peritoneal Mesothelial Cells. J. Am. Soc. Nephrol. 2001, 12, 2434-2441.

14. Witowski, J.; Jörres, A.; Korybalska, K.; Ksiazek, K.; Wisniewska-Elnur, J.; Bender, T.O.; Passlick-Deetjen, J.; Breborowicz, A. Glucose Degradation Products in Peritoneal Dialysis Fluids: Do They Harm? Kidney Int. 2003, 63, S148-S151.

15. Witowski, J.; Bender, T.O.; Gahl, G.M.; Frei, U.; Jörres, A. Glucose Degradation Products and Peritoneal Membrane Function. Perit. Dial. Int. 2001, 21, 201-205.

16. Vardhan, A.; Hutchison, A.J. Peritoneal Dialysis Solutions. In: Nissenson, A.R.; Fine, R.N. editors. Handbook of Dialysis Therapy. 5th ed. Elsevier; New York, NY, USA. 2017.

17. Hsu, Y.H.; Chen, Y.W.; Cheng, C.Y.; Lee, S.L.; Chiu, T.H.; Chen, C.H. Detecting the limits of the biological effects of far-infrared radiation on epithelial cells. Scientific Reports. 201, 9(1), 11586.

18. Henderson, T.A. Multi-watt near-infrared light therapy as a neuroregenerative treatment for traumatic brain injury. Neural Regeneration Research. 2016, 11(4), 563-565.

19. Leung, T.K. In Vitro and In Vivo Studies of the Biological Effects of Bioceramic (a Material of Emitting High Performance Far-Infrared Ray) Irradiation. Chin J Physiol. 2015, 58(3), 147-55.

20. Hamada, Y.; Teraoka, F.; Matsumoto, T.; Madachi, A.; Toki, F.; Uda, E.; Hase, R.; Takahashi, J.; Matsuura, N. Effects of far infrared ray on Hela cells and WI-38 cells. International Congress Series. 2003, 1255, 339- 341.

21. Ou, S.M.; Hu, F.H.; Yang, W.C.; Lin, C.C. Far-Infrared Therapy as a Novel Treatment for Encapsulating Peritoneal Sclerosis. Am. J. Gastroenterol. 2014, 109, 1957-1959.

22. Akasaki, Y.; Miyata, M.; Eto, H.; Shirasawa, T.; Hamada, N.; Ikeda, Y.; Biro, S.; Otsuji, Y.; Tei, C. Repeated Thermal Therapy Up-Regulates Endothelial Nitric Oxide Synthase and Augments Angiogenesis in a Mouse Model of Hindlimb Ischemia. Circ. J. 2006, 70, 463-470.

23. Yu, S.Y.; Chiu, J.H.; Yang, S.D.; Hsu, Y.C.; Lui, W.Y.; Wu, C.W. Biological Effect of Far-Infrared Therapy on Increasing Skin Microcirculation in Rats. Photodermatol. Photoimmunol. Photomed. 2006, 22, 78-86.

24. Chang, Y. The Effect of Far Infrared Radiation Therapy on Inflammation Regulation in Lipopolysaccharide-induced Peritonitis in Mice. SAGE Open Med. 2018, 6, 2050312118798941.

25. Inoue, S.; Kabaya, M. Biological activities caused by far-infrared radiation. Int J Biometeorol. 1989, 33(3), 145-50.

26. Oosterveld, F.G.; Rasker, J.J.; Floors, M.; Landkroon, R.; van Rennes, B.; Zwijnenberg, J.; van de Laar, M.A.; Koel, G.J. Infrared sauna in patients with rheumatoid arthritis and ankylosing spondylitis. A pilot study showing good tolerance, short-term improvement of pain and stiffness, and a trend towards long-term beneficial effects. Clin Rheumatol. 2009, 28(1), 29- 34.

27. Masuda, A.; Koga, Y.; Hattanmaru, M.; Minagoe, S.; Tei, C. The effects of repeated thermal therapy for patients with chronic pain. Psychother Psychosom. 2005, 74(5), 288-94.

28. Lee, D.; Seo, Y.; Kim, Y.W.; Kim, S.; Bae, H.; Choi, J.; Lim, I.; Bang, H.; Kim, J.H.; Ko, J.H. Far-infrared radiation stimulates platelet-derived growth factor mediated skeletal muscle cell migration through extracellular matrix-integrin signaling. Korean J Physiol Pharmacol. 2019, 23(2), 141-150.

29. Toyokawa, H.; Matsui, Y.; Uhara, J.; Tsuchiya, H.; Teshima, S.; Nakanishi, H.; Kwon, A.H.; Azuma, Y.; Nagaoka, T.; Ogawa, T.; Kamiyama, Y. Promotive effects of far-infrared ray on full-thickness skin wound healing in rats. Exp Biol Med (Maywood). 2003, 228(6), 724-9.

30. Capon, A.; Mordon, S. Can thermal lasers promote skin wound healing? Am J Clin Dermatol. 2003, 4(1), 1-12.

31. Hsu, Y.H.; Lin, Y.F.; Chen, C.H.; Chiu, Y.J.; Chiu, H.W. Far infrared promotes wound healing through activation of Notch1 signaling. J Mol Med (Berl). 2017, 95(11), 1203-1213.

32. Chiu, H.W.; Chen, C.H.; Chang, J.N.; Chen, C.H.; Hsu, Y.H. Farinfrared promotes burn wound healing by suppressing NLRP3 inflammasome caused by enhanced autophagy. J Mol Med (Berl). 2016, 94(7), 809-19.

33. Ikeda, Y.; Biro, S.; Kamogawa, Y.; Yoshifuku, S.; Eto, H.; Orihara, K.; Yu, B.; Kihara, T.; Miyata, M.; Hamasaki, S. Repeated Sauna Therapy Increases Arterial Endothelial Nitric Oxide Synthase Expression and Nitric Oxide Production in Cardiomyopathic Hamsters. Circ. J. 2005, 69, 722-729.

34. Lin, C.C.; Yang, C.C.; Hsiao, L.D.; Chen, S.Y.; Yang, C.M. Heme Oxygenase-1 Induction by Carbon Monoxide Releasing Molecule-3 Suppresses Interleukin-1 $\beta$-Mediated Neuroinflammation. Front. Mol. Neurosci. 2017, 10, 387. 
35. Chen, S.C.; Lee, M.Y.; Huang, J.C.; Kuo, I.C.; Mai, H.C.; Kuo, P.L.; Chang, J.M.; Hwang, S.J.; Chen, H.C. Association of Far-Infrared Radiation Therapy and Ankle-Brachial Index of Patients on Hemodialysis with Peripheral Artery Occlusive Disease. International Journal of Medical Sciences. 2016, 13(12), 970-976.

36. Lin, C.C.; Liu, X.M.; Peyton, K.; Wang, H.; Yang, W.C.; Lin, S.J.; Durante, W. Far Infrared Therapy Inhibits Vascular Endothelial Inflammation via the Induction of Heme Oxygenase-1. Arteriosclerosis, Thrombosis, and Vascular Biology. 2008, 28, 739-745.

37. Su, L.H.; Wu, K.D.; Lee, L.S.; Wang, H.; Liu, C.F. Effects of far infrared acupoint stimulation on autonomic activity and quality of life in hemodialysis patient. The American Journal of Chinese Medicine. 2009, 37(2), $215-226$.

38. Jeong, Y.M.; Cheng, X.W.; Lee, S.; Lee, K.H.; Cho, H.; Kang, J.H.; Kim, W. Preconditioning with far-infrared irradiation enhances proliferation, cell survival, and migration of rat bone marrow-derived stem cells via CXCR4-ERK pathways. Sci Rep, 2017, 7(1), 13718.

39. Liao, B.; Zhou, H.; Liang, H.; Li, C. Regulation of ERK and AKT pathways by hepatitis B virus X protein via the Notch1 pathway in hepatocellular carcinoma. Int J Oncol. 2017, 51(5), 1449-1459.

40. De Luca, A.; Maiello, M.R.; D'Alessio, A.; Pergameno, M.; Normanno, N. The RAS/RAF/MEK/ERK and the PI3K/AKT signalling pathways: role in cancer pathogenesis and implications for therapeutic approaches. Expert Opin Ther Targets. 2012, 16 (Suppl 2), S17-27.

41. Lin, C.C.; Chung, M.Y.; Yang, W.C.; Lin, S.J.; Lee, P.C. Length polymorphisms of hemeoxygenase-1 determine the effect of far-infrared therapy on the function of arteriovenous fistula in hemodialysis patients: a novel physicogenomic study. Nephrology Dialysis Transplantation. 2013, 28(5), 1284-1293.

42. Salm, D.C.; Belmonte, L.A.O.; Emer, A.A.; Leonel, L.D.S.; de Brito, R.N.; da Rocha, C.C.; Martins, T.C.; Dos Reis, D.C.; Moro, A.R.P.; Mazzardo-Martins, L.; Kviecinski, M.R.; Bobinski, F.; Salgado, A.S.I.; Cidral-Filho, F.J.; Martins, D.F. Aquatic exercise and Far Infrared (FIR) modulates pain and blood cytokines in fibromyalgia patients: A double-blind, randomized, placebo-controlled pilot study. J Neuroimmunol. 2019, 337, 577077.

43. Hsu, Y.H.; Chen, Y.C.; Chen, Y.W.; Chiu, T.H.; Kuo, Y.T.; Chen, C.H. Far-infrared radiation prevents decline in $\beta$-cell mass and function in diabetic mice via the mitochondria-mediated Sirtuin1 pathway. Metabolism. 2020, 104, 154143.

44. Chang, J.C.; Wu, S.L.; Hoel, F.; Cheng, Y.S.; Liu, K.H.; Hsieh, M.; Hoel, A.; Tronstad, K.J.; Yan, K.C.; Hsieh, C.L.; Lin, W.Y.; Kuo, S.J.; Su, S.L.; Liu, C.S. Far-infrared radiation protects viability in a cell model of Spinocerebellar Ataxia by preventing polyQ protein accumulation and improving mitochondrial function. Sci. Rep. 2016, 6, 30436.

45. Minutti, C.M.; Knipper, J.A.; Allen, J.E.; Zaiss, D.M. Tissue-specific contribution of macrophages to wound healing. Semin. Cell Dev. Biol. 2017, 61, 3-11.

46. Van Dyken, S.J.; Locksley, R.M. Interleukin-4- and interleukin-13-mediated alternatively activated macrophages: roles in homeostasis and disease. Annu. Rev. Immunol. 2013, 31, 317-343.

47. Pearce, E.J.; MacDonald, A.S. The immunobiology of schistosomiasis. Nat. Rev. Immunol. $2002,2,499-511$.

48. Fairfax, K.; Nascimento, M.; Huang, S.C.; Everts, B.; Pearce, E. J. Th2 responses in schistosomiasis. Seminars Immunopathol. 2012, 34, 863-871.

49. Dong, C. Cytokine Regulation and Function in T Cells. Annu Rev Immunol. 2021, 39, 51-76.

50. Wang, X.; Lin, X.; Zheng, Z.; Lu, B.; Wang, J.; Tan, A.H.; Zhao, M.; Loh, J.T.; Ng, S.W.; Chen, Q.; Xiao, F.; Huang, E.; Ko, K.H.; Huang, Z.; Li, J.; Kok, K.H.; Lu, G.; Liu, X.; Lam, K.P.; Liu, W.; Zhang, Y.; Yuen, K.Y.; Mak, T.W.; Lu, L. Host-Derived Lipids Orchestrate Pulmonary $\gamma \delta \mathrm{T}$ Cell Response to Provide Early Protection Against Influenza Virus Infection. Nat Commun. 2021, 12(1), 1914.

51. Lai, C.C.; Fang, H.C.; Mar, G.Y.; Liou, J.C.; Tseng, C.J.; Liu, C.P. Post-angioplasty far infrared radiation therapy improves 1-year angioplasty-free hemodialysis access patency of recurrent obstructive lesions. European Journal of Vascular and Endovascular Surgery. 2013, 46(6), 726-732.

52. Lin, C.C.; Chung, M.Y.; Yang, W.C.; Lin, S. J.; Lee, P.C. Length polymorphisms of heme oxygenase-1 determine the effect of far-infrared therapy on the function of arteriovenous fistula in hemodialysis patients: a novel physicogenomic study. Nephrology Dialysis Transplantation. 2013, 28(5), 1284-1293.

53. Lin, C.C.; Yang, W.C.; Chen, M.C.; Liu, W.S.; Yang, C.Y.; Lee, P.C. Effect of far infrared therapy on arteriovenous fistula maturation: an open-label randomized controlled trial. American Journal of Kidney Diseases. 2013, 62(2), 304-311. 
54. Fu, Q.; Li, Z.; Ye, J.; Li, Z.; Fu, F.; Lin, S.L.; Chang, C.A.; Yang, H.; Song, J. Magnetic targeted near-infrared II PA/MR imaging guided photothermal therapy to trigger cancer immunotherapy. Theranostics. 2020, 10(11), 4997-5010.

55. Huang, P.H.; Chen, J.W.; Lin, C.P.; Chen, Y.H.; Chen, Y.H.; Wang, C.H.; Leu, H.B.; Lin, S.J. Far infra-red therapy promotes ischemia-induced angiogenesis in diabetic mice and restores high glucose-suppressed endothelial progenitor cell functions. Cardiovasc Diabetol. 2012, 11, 99-99.

56. Nicola, R.S.; Jason, J.A. Role of C-Reactive Protein at Sites of Inflammation and Infection. Front Immuno. 2018, 9 , 754.

57. Choi, G.B.; Yim, Y.S.; Wong, H.; Kim, S.; Kim, H.; Kim, S.V.; Hoeffer, C.A.; Littman, D.R.; Huh, J.R. Thematernal interleukin-17a pathway in mice promotes autism-like phenotypes in offspring. Science. 2016, 351, 933-939. 